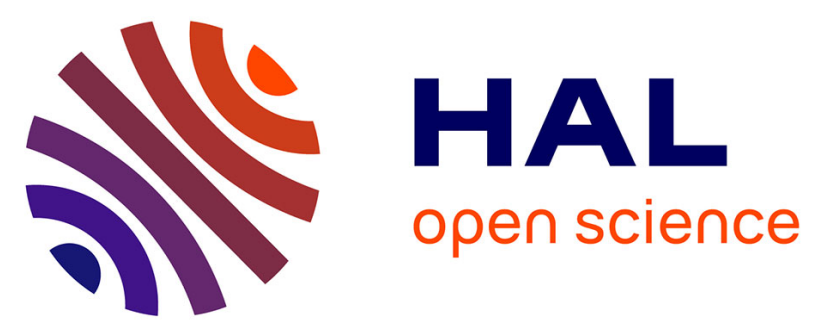

\title{
Indirect analysis of the position of a doubly-salient variable-reluctance machine by optimal synchronous demodulation of PWM signals
}

Philippe Laurent, Bernard Multon, Emmanuel Hoang, Mohamed Gabsi

\section{- To cite this version:}

Philippe Laurent, Bernard Multon, Emmanuel Hoang, Mohamed Gabsi. Indirect analysis of the position of a doubly-salient variable-reluctance machine by optimal synchronous demodulation of PWM signals. 1995 Canadian Conf. on Electrical and Comp. Engineering, Sep 1995, MONTREAL, Canada. pp.264-267. hal-00674016

\author{
HAL Id: hal-00674016 \\ https://hal.science/hal-00674016
}

Submitted on 24 Feb 2012

HAL is a multi-disciplinary open access archive for the deposit and dissemination of scientific research documents, whether they are published or not. The documents may come from teaching and research institutions in France or abroad, or from public or private research centers.
L'archive ouverte pluridisciplinaire HAL, est destinée au dépôt et à la diffusion de documents scientifiques de niveau recherche, publiés ou non, émanant des établissements d'enseignement et de recherche français ou étrangers, des laboratoires publics ou privés. 


\author{
P. LAURENT, B. MULTON, E. HOANG, M. GABSI \\ L.E.S.i.R URA CNRS D1375 \\ ENS Cachan, 61 Avenue du Pdt. Wilson 94235 CACHAN CEDEX FRANCE \\ Tel: (33) (1) $47402111 \quad$ Fax:(33) (1) 47402199 \\ Internet: philippe.LAURENT@lesir.ens-cahan.fr
}

\begin{abstract}
Résume: Cet article propose une méthode originale d'utilisation des signaux du convertisseur de puissance pour la détermination de la position du rotor d'une machine a réluetance variable en vue de son autopilotage. Ces signaux à haute fréquence $(20 \mathrm{kHz})$ fournissent la porteuse de scrutation et de mesure des paramètres du modèle de la phase de la machine. Une optimisation de la démodulation synchrone de cette porteuse permet de réduire considérablement l'influence de la saturation sur la valeur de ces paramètres tout en augmentant leur variation relative vis a vis de la position du rotor.
\end{abstract}

\section{INTRODUCTION}

Dans les Machines à Réluctance Variable à Double Saillance (MRV), le couple est généré par l'alimentation successive de chacune des phases statorique en fonction de la position du rotor. Ce principe d'autopilotage de la machine nécessite dans la plupart des cas de recourir à l'utilisation d'un capteur de position rotorique (optique dans le cas général) ce qui nuit à la robustesse ainsi qu'au prix de revient de l'entrainement. Pour remédier à l'utilisation d'un tel capteur, de nombreux auteurs ont proposé des solutions rechniques de remplacement que l'on peut classer en deux principales familles distinctes:

La première famille de processus repose sur la mesure de la position rotorique via la scrutation d'une phase qui n'est pas en cours d'alimentation par le convertisseur de puissance:

- mesure de l'inductance propre par démodulation d'amplitude [3] [7], de fréquence [4] ou de phase.

- injection d'une impulsion de tension de scrutation puis mesure du correspondant. [5], [10]

- Mesure des tensions induites par la phase en cours d'alimentation dans une autre phase ce qui nécessite un couplage magnétique significatif [6]

Ces méthodes sont utilisables dans le cas d'une alimentation en tension ou courant mais la durée de la fenêtre de scrutation diminue avec la vitesse et le remps de démagnétisation de chacune des phases à scruter. Un second problème réside dans l'apparition de la saturation magnétique lors de la mesure de l'inductance.

La seconde famille de processus repose sur la mesure des courants et tensions imposées par le convertisseur dans une phase alimentee:

- Mesure de la position par analyse de la réponse en courant [9] mais des non-linéarites dépendantes de la force électromotrice interne et de la saturation magnétique apparaissent.

- Mesure du $\frac{d l}{d t}$ dans le cas d'une alimentation en tension [2] et prise en compte de la force électromotrice interne (des problèmes de précision apparaissent alors à très basse vitesse).

- Méthodes sophistiquée basees sur la reconstruction des signaux électriques de la machines grâce à un modèle temps réel[11], [12]. Ceci nécessite un système informatique puissant et rapide qui ne prend d'ailleurs pas en compte efficacement les couplages magnétiques et la saturation magnétique (nécessité d'un modèle très complexe).

\section{MODELISATION CLASSIQUE D'UNE MRV}

Nous avons cherché à mesurer l'ensemble des paramètres du modèle electrique d'une phase en cours d'alimentation puis de combiner la mesure de ces parametres en vue de minimiser l'influence de la saturation magnétique sur l'estimation de la position. La machine d'étude ainsi que le convertisseur de puissance sont présentés en fig. 1. Il s'agit d'une machine à réluctance variable avec un double stator monophasé et dotée de 4 dents rotoriques (1 paire de pôles par phases).
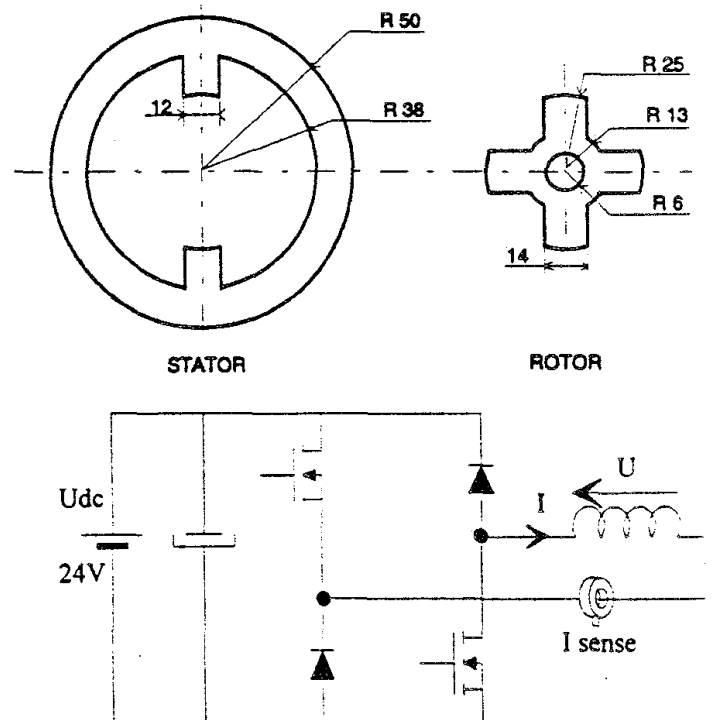

Fig. 1: Machine à Réluctance Variable double stator monophasé associée à son convertisseur demi-pont asymétrique (dimensions données en $\mathrm{com}$ ).

Il est possible d'adopter un schéma de type transformateur monophasé pour modéliser le comportement de la phase soumis aux signaux du convertisseur de puissance (Fig. 2). Sur certe figure, Ro, Lf, Rsi, Rri représentent respectivement la résistance ohmique, l'inductance de fuite, les résistances équivalentes aux pertes fer dans la culasse stator et le reste de la machine(dents rotor et stator). Lorsque l'on soumet la phase à une tension altemative cartée $\left(-\mathrm{U}_{\mathrm{dc}} /-\mathrm{U}_{\mathrm{dc}}\right)$ l'amplitude de l'induction magnétique $B$ reste constante dans la culasse mais varie suivant la position dans les dentures. 


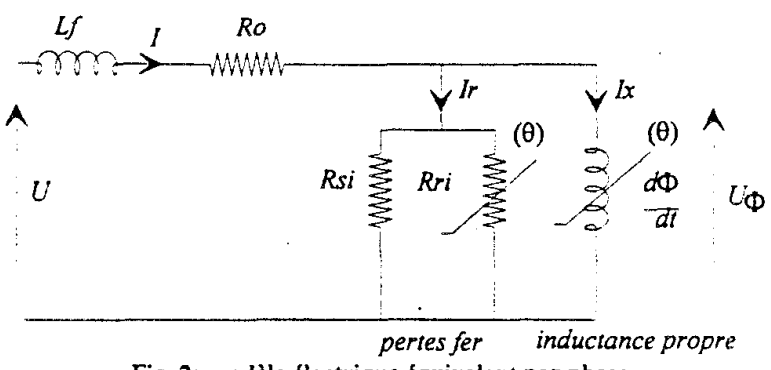

Fig. 2: modèle électrique équivalent par phase.

L'équation de magnétisation correspondante peut donc s'écrire comme suit:

$\mathrm{U}=\mathrm{L}_{\mathrm{f}} \cdot \frac{\mathrm{dl}}{\mathrm{dt}}+\mathrm{R}_{\mathrm{o}} \cdot 1+\frac{\mathrm{d} \Phi}{\mathrm{dt}}$ et $\frac{\mathrm{d} \Phi}{\mathrm{dt}}=\frac{\mathrm{R}_{\mathrm{si}} \cdot \mathrm{R}_{\mathrm{ri}}}{\mathrm{R}_{\mathrm{si}}+\mathrm{R}_{\mathrm{ri}}} \cdot I_{\mathrm{r}}$

Avec la fréquence de découpage choisie pour cette application $(20 \mathrm{kHz})$, on peut considérer que Lf et Ro ont peu d'influence vis à vis de la tension induite dans l'enroulement et pourront être négligées ( sinon on peut utiliser une spire de mesure pour determiner $\left.U_{\Phi}\right)$.

Pour modéliser le comportement de la phase en haute fréquence, on peut limiter la complexité du schéma aux seuls éléments magnétiques significatifs: Rsi, Rri et l'inductance propre et le décliner en deux variantes mathématiquement identifiables. Un modèle parallèle (fig. 3a) et un modèle série (fig. 3b) .:

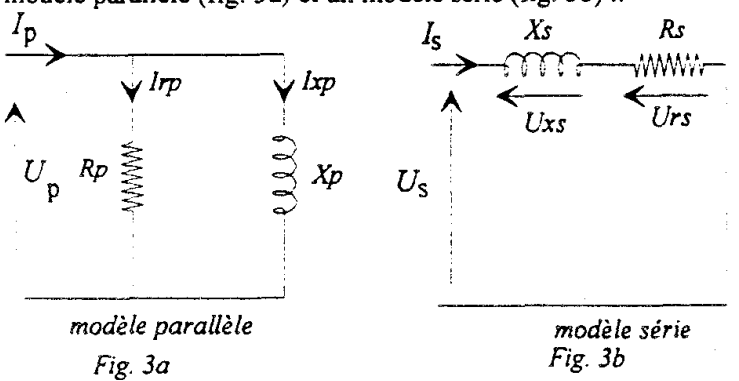

Fig. 3: notations employées pour le modèle électrique parallèle et série.

\section{RELATION ENTRE MODELE PARALLELE ET SERIE .}

Il est très facile d'exprimer les éléments du modèle série en fonction de ceux du modèle parallèle. On aboutit ainsi aux deux relations suivantes:

$$
R_{s}=\frac{R_{p} \cdot X_{p}^{2}}{R_{p}^{2}+X_{p}^{2}} \quad \text { et } \quad X_{s}=\frac{X_{p} \cdot R_{p}^{2}}{R_{p}^{2}+X_{p}^{2}}
$$

\section{A. MODELEPARALLELE}

En utilisant une MLI bipolaire due au découpage comme porteuse de tension de scrutation et en se limitant à la mesure du premier harmonique, il est possible de déterminer pratiquement le terme $\mathrm{Rp}$ et Xp désignant respectivement le terme réel et imaginaire du schéma équivalent parallèle en effectuant une démodulation synchrone de l'ondulation de courant due au découpage.

Dans ce cas l'onde de tension constitue la référence de phase et en fonction de l'angle $\alpha$ d'échantillonnage par rapport au passage par zéro de l'onde du fondamental de tension, on peut écrire dans le cas général:

$$
I_{p}(\alpha)=\left|U_{p}\right| \cdot\left(\frac{\sin (\alpha)}{R_{p}}+\frac{\sin \left(\alpha-\frac{\pi}{2}\right)}{X_{p}}\right)
$$

11 est possible de normaliser cette expression vis a vis de $\left|U_{p}\right|$ de manière à s'affranchir de la valeur du rappor cyclique de conduction de l'onduleur. Dans ce cas on dissocie $R_{p}$ et $X_{p}$ en choisissant convenablement $\alpha$. On aboutit ainsi à la mesure du courant actif normalise $I_{\text {rpn }}$ et du courant réactif normalisé $\mathrm{l}_{\mathrm{xpn}}$ :

$$
l_{x p n}=\frac{I_{p}(\pi / 2)}{\left|U_{p}\right|}=\frac{1}{R_{p}} \text { et } I_{x p n}=\frac{I_{p}(\pi)}{\left|U_{p}\right|}=\frac{1}{X_{p}}
$$

Pour étudier l'influence de la saturation magnétique sur l'évolution de ces deux paramètres nous avons alimenté une phase de la machine avec une composante sinusoïdale de courant à $20 \mathrm{kHz}$ en plus d'une polarisation en courant continu variable. Les paramètres Rp et Xp qui dépendent de la position du rotor sont mesurés à $20 \mathrm{kHz}$ uniquement à l'aide d'un wattmètre électronique. Les résultats de la mesure concernant Irpn et Ixpn sont présentés respectivement en fig. 4 et fig. 5 .

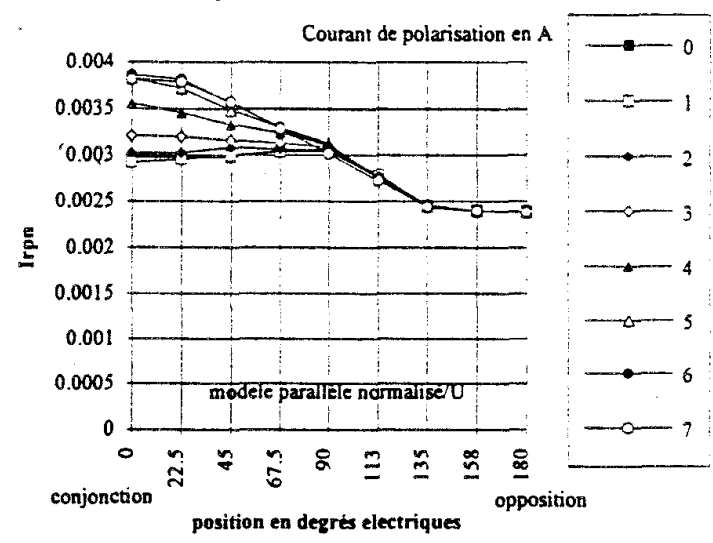

Fig. 4: mesure de Irpn (composante active) \20kHz pour différents niveaux de courants de polarisation du circuit magnetique $\left(\alpha=90^{\circ}\right)$

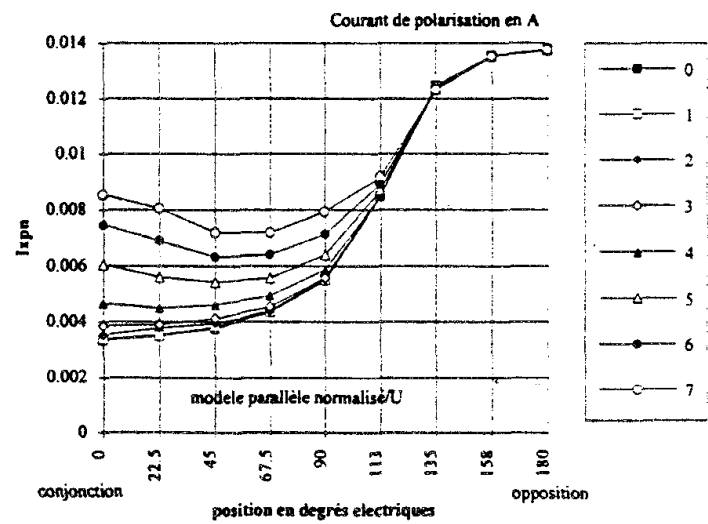

Fig.5: mesure de Ixpn (composante réactive) à $20 \mathrm{kHz}$ pour différents niveaux de courants de polarisation du circuit magnétique $\left(\alpha=180^{\circ}\right)$

On peut constater que la saturation magnétique qui apparaît dès les faibles niveaux de polarisation $(3 \mathrm{~A})$ perturbe la mesure des paramètres réel (Irpn) et réactif (Ixpn) et fausse l'estimation de la position. Ce phénomène est bien connu en ce qui concerne le parametre réactif car il se traduit par une diminution de l'inductance incrémentale mais aussi par une diminution des pertes fer de découpage comme le montre la figure 4 . Il ne semble donc pas à priori possible d'obtenir la position rotorique par la seule mesure de l'un des deux paramètres du schéma équivalent décrit en fig. 3. Nous avons donc cherché à optimiser l'angle d'échantillonnage $\alpha$ de la démodulation synchrone de manière à minimiser l'influence de cette saturation sur notre mesure.

Un autre problème réside dans la sensibilité relative de la mesure vis à vis de la position $\left(\frac{d l_{p n}}{d \theta}\right)$. Une optimisation de $\alpha$ est encore possible pour combiner l'évolution de $\mathrm{Rp}$ et $\mathrm{Xp}$ de manière à obtenir une meilleure sensibilité relative de la mesure de position. 
$L$ 'optimum est atteint pour $\alpha=80^{\circ}$ et les résultats sont présentés en fig. 6

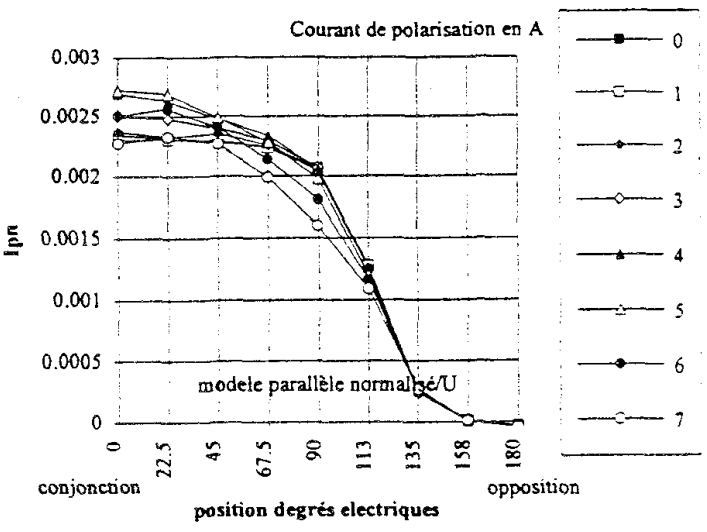

Fig.6: évolution $\operatorname{lp}(\alpha)$ pour differents courants de polarisation après optimisation de l'angie d'échantillonnage $=80^{\circ}$.

On peut ainsi constater que l'influence de la saturation magnétique est considérablement réduite ce qui permet d'obtenir une estimation de la position relativement fidèle quelque soit le régime de polarisation en courant. D'autre part, la sensibilité globale de la mesure est fortement augmenté puisque l'opposition correspond à un courant démodulé nul.

\section{B. MODELE SERIE}

Cette fois, la mesure de Rs et Xs s'effectue en démodulant le signal fondamental de la composante de tension due au découpage avec un angle $\beta$ d'échantillonnage par rapport au passage par zéro du fondamental de courant. L'équation générale de la tension démodulée est la suivante:

$$
U_{s}(\beta)=\left|I_{s}\right| \cdot\left(R_{s} \cdot \sin (\beta)+X_{s} \cdot \sin \left(\beta-\frac{\pi}{2}\right)\right)
$$

Une démarche de normalisation par rapport à $\left|I_{s}\right|$ peut être adoptée pour normaliser l'expression et s'affranchir du rapport cyclique de conduction de l'onduleur. Dans ce demier cas, en adoptant respectivement $\beta=90^{\circ}$ et $\beta=180^{\circ}$ il est possible d'isoler directement Rs et Xs. Comme dans le modèle parallèle, nous avons cherché à optimiser la mesure de tension démodulée de manière à la rendre insensible à la saturation. Un résultat intéressant est présenté en figure 7 pour un angle d'échantillonnage de $215^{\circ}$

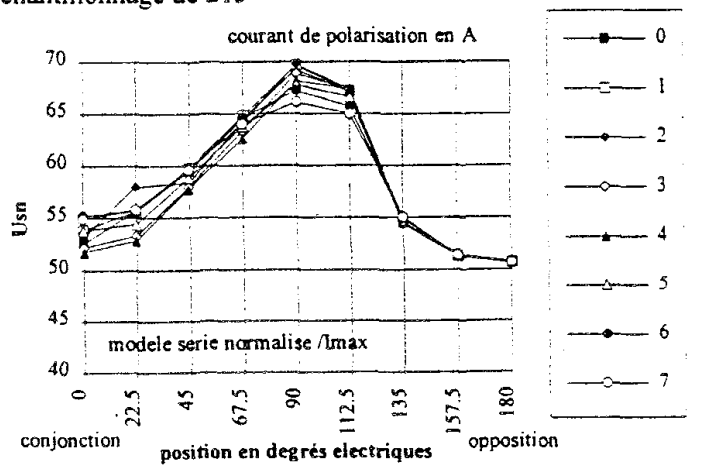

Fig. 7: évolution Us( $\beta)$ pour différents courants de polarisation après optimisation de l'angle d'echantillonnage $=215^{\circ}$ 。

On peut remarquer que le maximum de déviation du signal démodulé ne correspond plus à une position de conjonction mais se trouve localisé de manière symétrique entre opposition et conjonction. Cependant, pour ce type de machine le résultat d'optimisation donne de meilleurs résultats avec la modélisation par schéma parallèle.

Pour en terminer entre les relations qui lient les deux modèles nous avons étudié une normalisation homogène des mesures de Us et Ip, c'est à dire que le courant $I p(\alpha)$ est normalisé par rapport à $\left|\mathrm{I}_{\mathrm{p}}\right|$, de même pour Us $(\beta)$ qui est normalisé par rapport à $\left|\mathrm{U}_{\mathrm{s}}\right|$. On peut écrire dans les deux cas précédents les relations correspondant aux courant et tension démodulés avec ce type de normalisation respectivement pour les schémas parallèle et série:

$$
\begin{aligned}
& I_{p a h}(\alpha)=\frac{I_{p}(\alpha)}{\left|I_{p}\right|}=\frac{\sin \alpha}{R_{p} \cdot \sqrt{\frac{1}{R_{p}^{2}}+\frac{1}{X_{p}^{2}}}}+\frac{\sin (\alpha-\pi / 2)}{X_{p} \cdot \sqrt{\frac{1}{R_{p}^{2}}+\frac{1}{X_{p}^{2}}}} \\
& U_{\operatorname{sanh}}(\beta)=\frac{U_{s}(\beta)}{\left|U_{s}\right|}=\frac{R_{s} \cdot \sin \beta}{\sqrt{R_{s}^{2}+X_{s}^{2}}}+\frac{X_{s} \cdot \sin (\beta-\pi / 2)}{\sqrt{R_{s}^{2}+X_{s}^{2}}}(7)
\end{aligned}
$$

Dans ce cas on montre à l'aide de l'équation (2) que les deux mesures effectuées sont rigoureusement équivalentes quelque soit $\alpha=\beta$. Cette demière normalisation n'apporte pas de résultats intéressant dans notre exemple mais on peut envisager d'essayer d'optimiser l'angle d'échantillonnage pour minimiser l'influence de la saturation pour d'autres configurations de machines. D'une manière un peu pus générale, on a pu observer que l'angie d'optimisation dépendait du niveau de courant de polarisation à partir duquel intervenait la saturation. Cette angle sera voisin de $90^{\circ}$ pour une machine fortement saturable, et voisin $180^{\circ}$ dans le cas d'une machine à entrefer important. La répartition de courant Ipn entre Rpn et Xpn fixe à priori la valeur de cet angle optimale.

\section{SYNOPTIQUE DE LA COMMANDE SANS CAPTEUR}

Pour obtenir et mesurer distinctement le premier harmonique des composantes de découpage de la tension et du courant du découpage à partir des signaux d'alimentation il est nécessaire de disposer de deux filtres passe-bande rigoureusement identiques de manière à éliminer toute erreur de déphasage entre les deux signaux à démoduler. La solution la plus simple est d'opérer à l'aide de filtres à capacités commutées séquencés par le micro contrôleur qui gère aussi le découpage (voir fig. 8).Puis une démodulation synchrone, gérée par les timers du dsp et opérant avec l'angle $\alpha$ (ou $\beta$ pour le modèle série) issus de l'optimisation, fournit le courant normalisé image de la position (dans le cas du modèle

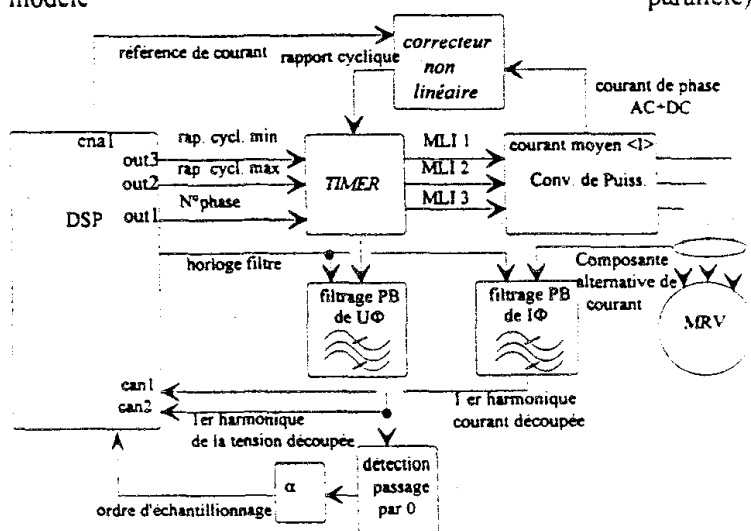

Fig. 8: synoptique de la commande sans capteur; démodulation synchrone de la porteuse généré par le découpage de puissance.

Par comparaison à une valeur de référence, on ordonne le passage de l'alimentation à la phase suivante ce qui permet un fonctionnement en boucle fermée. 


\section{RESULTATS EXPERIMENTAUX}

Nous présentons en fig. 9 un exemple de signal image du premier harmonique de l'ondulation (MLI) du courant dans la phase, obtenue après filtrage passe bande (ch2) ainsi que le signal lpn obtenu après démodulation synchrone avec l'angie d'échantillonnage optimisé $\alpha$ (ch1) à une vitesse de $7500 \mathrm{tr} / \mathrm{min}$

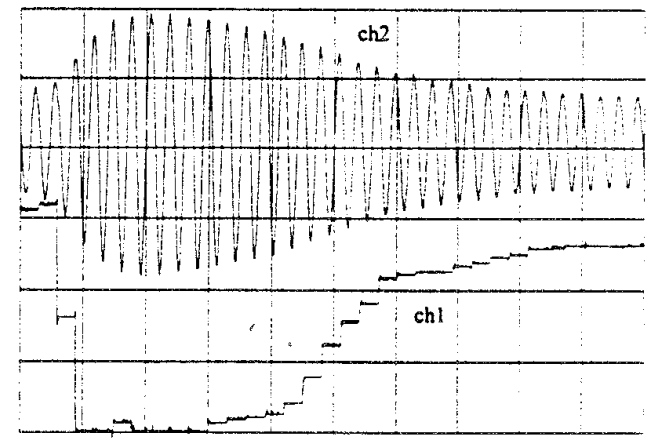

time base: 200us/div

Fig. 9: exemple de signal image du $1^{\text {er }}$ harmonique de courant de découpage (ch2) et mesure de Ipn après démodulation synchrone (chl) suivant l'angle d'échantillonnage $\alpha$ optimisé.

La fig. 9 se déduit de la courbe théorique (fig.6) par symétrie verticale autour de l'axe d'opposition $\left(\theta=180^{\circ}\right)$ car le déplacement angulaire en mode moteur s'effectue depuis l'opposition vers la conjonction. Le point de référence de commutation doit être choisi de manière à propager l'alimentation vers la phase prète à entrer en conduction dès que la phase en cours d'alimentation anteint sa conjonction magnétique. $\mathrm{Ce}$ point de référence doit donc correspondre à la conjonction.

Un algorithme plus complexe permet de prendre comme point de référence de mesure non plus la conjonction mais une position intermédiaire entre l'opposition et la conjonction. On pourra remarquer que cette position correspondant à un angle électrique de $90^{\circ}$ sur la figure 6 permet de mieux s'affranchir de la saturation. D'autre part, cette position constitue un point de variation relative maximale de la mesure $\operatorname{lpn}(\alpha)$ vis à vis de la position rotorique. Un intégrateur numérique mesure le temps écoulé depuis le début de la magnétisation (opposition) jusqu'au croisement avec cette valeur de référence et décompte à nouveau jusqu'à zéro. En fin de décomptage (au voisinage de la conjonction $180^{\circ}$ ) le système ordonne l'alimentation de la phase suivante et ainsi de suite. L'algorithme agit dans ce cas comme une boucle à verrouillage de phase et la commutation d'alimentation de la phase correspond à une estimation de la position de conjonction à partir d'une mesure plus sensible de la position à $90^{\circ}$. L'autre intérêt de cette méthode est quelle permet de régler numérique l'instant de commutation d'alimentation par rapport à la position atteinte par l'intégrateur numérique indépendamment de la référence de mesure des signaux démodulés. Un réglage de l'angle d'avance à la mise en conduction est donc ainsi réalisable de manière très précise. La seule contrainte reste de pouvoir maintenir au moins une commutation ce qui exclus un régime de pleine onde en haute vitesse mais cette limitation n'impose qu'un léger surdimenssionnement en tension de l'onduleur.

\section{CONCLUSION}

Une nouvelle méthode d'autopilotage de MRV utilisant le découpage du convertisseur comme porteuse de détection et de mesure des éléments du schéma équivalent électrique de la machine a été décrite. Une étude d'optimisation de la démodulation synchrone de cette porteuse de détection montre sur cet exemple une amélioration sensible de la mesure de la position du rotor vis à vis de la saturation magnétique et de la sensibilité relative de la mesure de position. II apparaît ainsi quelques avantages inhérent au processus:

- pas d'électronique supplémentaire d'injection de porteuse.

- pas de problèmes d'immunité aux bruits de commutation du convertisseur grâce au filtrage passe bande à capacités commutées.

- possibilité d'ajuster finement l'angie d'avance à l'allumage de manière à désexciter la machine en régime de haute vitesse.

\section{BIBLIOGRAPHIE}

[1] W.F. RAY L.H. AZI-BAHADLY

"Sensorless Methods for Determining the Rotor Position of Switched Reluctance Motors."

Proceedings of EPE 93, Vol 6, 1993, pp 7.13

[2] P. CARNE KJAER

"A New Indirect Rotor Position Detection Method for Switched Reluctance Motors."

Proceedings of ICEM 94, Vol 2, 1994, pp 555-560

[3] M. EHSANI, I.HUSAN, A.B. KULKARNI

"Elimination of Position and Current sensor in Switched Reluctance Motor Drives."

IEEE Trans I.A., Vol 28, n²1, 1992, pp 128-135

[4] M. EHSANI, S. MAHAJAN, KR. RAMANI, I.HUSAIN

New Modulation Encoding Techniques for Indirect Rotor Position Sensing in Switched Reluctance Motors."

Proceedings of IEEE IAS 92, Vol 1, 1992, pp 430-438

[5] N.H. MVUNGI, J.M. STEPHENSON

"Accurate Sensorless Rotor Position Detection in a S. R. Motor."

Proceedings of EPE 91, Vol 1, 1991, pp 390-393

[6] M. EHSANI, I HUSAIN

"Rotor Position Sensing in Switched Reluctance Motor Drives bv measuring Mutualiy Induced Voltage."

Proceedings of IEEE IAS Houston 92, Vol 1, 1992 pp $422-429$

[7] P. LAURENT, M. GABSI. B. MULTON

"Sensorless Position Analysis using Resonant Method for Switched Reluctance Motors."

Proceedings of IEEE LAS Toronto 93, Vol I 1993, pp 687.694

[8] M. EGAN, M.B. HARRINGTON, J.M.D. MURPHY

"PWM based Position Sensorless Control of Variable Reluctance Motor Drives."

Proceddings of EPE 91, Vol 4, 1991, pp 24-29

(9) P. ACARNELEY, R. HILL, C. HOOPER

"Detection of Rotor Position in Stepping And Switched Reluctance Motors by Monitoring of Current Waveforms".

IEEE Trans. 1.A. Vol 28, $\pi^{\circ} 6$, Nov/Dec 1992, pp 133-142.

[10] S.R. MACMINN, W. J. RZESOS, P.M. SZCZESNY, T.M. JAHNS "Application of Sensor Integration Techniques in Switched Reluctance Motor Drives".

IEEE Trans. I.E. Vol 28, n6, Nov/Dec 1992, pp 1339-1343.

[11] J.H. LANG。A. LUMSDAINE

"State Observers for Variable Reluctance Motor".

IEEE Trans. on I.E., Vol 37, n², 1990, pp 133-142.

[12] C. ELMAS, H. ZELAYA de la PARRA

"Position Sensorless Operation of a Switched Reluctance Drive Based on a State Observer".

Proceedings of EPE 93 Brighton, Vol 6, 1993, pp 82-87

[13] T.J.E. MILLLER, J.T. BARS, M. EHSANI

"Robuts Torque Control of Switched Reluctance Motors Without Shaft Sensor". IEEE Trans on I.E., Vol IE33, n³. 1986, pp 212-216 